\title{
BMJ open Structural determinants of food insufficiency, low dietary diversity and BMI: a cross-sectional study of HIV- infected and HIV-negative Rwandan women
}

\author{
Nicole Sirotin, ${ }^{1}$ Donald Hoover, ${ }^{2}$ C J Segal-Isaacson, ${ }^{3}$ Qiuhu Shi, ${ }^{4}$ \\ Adebola Adedimeji, ${ }^{5}$ Eugene Mutimura, ${ }^{6}$ Mardge Cohen, ${ }^{7}$ Kathryn Anastos ${ }^{8}$
}

To cite: Sirotin N, Hoover D, Segal-Isaacson CJ, et al. Structural determinants of food insufficiency, low dietary diversity and BMI: a cross-sectional study of HIV-infected and HIV-negative Rwandan women. BMJ Open 2012;2: e000714. doi:10.1136/ bmjopen-2011-000714

- Prepublication history for this paper is available online. To view these files please visit the journal online (http:// dx.doi.org/10.1136/ bmjopen-2011-000714)

Received 7 December 2011 Accepted 6 March 2012

This final article is available for use under the terms of the Creative Commons Attribution Non-Commercial 2.0 Licence; see http://bmjopen.bmj.com

For numbered affiliations see end of article.

Correspondence to Nicole Sirotin; nis9066@med.cornell.edu

\section{ABSTRACT}

Objectives: In Sub-Saharan Africa, the overlapping epidemics of undernutrition and HIV infection affect over 200 and 23 million people, respectively, and little is known about the combined prevalence and nutritional effects. The authors sought to determine which structural factors are associated with food insufficiency, low dietary diversity and low body mass index (BMI) in HIV-negative and HIV-infected SubSaharan women.

Study design: Cross-sectional analysis of a longitudinal cohort.

Setting: Community-based women's organisations.

Participants: 161 HIV-negative and 514 HIV-infected Rwandan women.

\section{Primary and secondary outcome}

measures: Primary outcomes included food insufficiency (reporting 'usually not' or 'never' to 'Do you have enough food?'), low household dietary diversity (Household Dietary Diversity Score $\leq 3$ ) and BMI $<18.5\left(\mathrm{~kg} / \mathrm{m}^{2}\right)$. The authors also measured structural and behavioural factors including: income, household size, literacy and alcohol use.

Results: Food insufficiency was prevalent $(46 \%)$ as was low dietary diversity (43\%) and low BMI (15\%). Food insufficiency and dietary diversity were associated with low income (adjusted odds ratio $(\mathrm{aOR})=2.14(95 \% \mathrm{Cl} 1.30$ to 3.52) $\mathrm{p}<0.01)$, $(\mathrm{aOR}=6.51(95 \% \mathrm{Cl} 3.66$ to 11.57$) \mathrm{p}<0.001)$, respectfully and illiteracy $(\mathrm{aOR}=2.00(95 \% \mathrm{Cl} 1.31$ to 3.04) $p<0.01),(a \mathrm{R}=2.10(95 \% \mathrm{Cl} 1.37$ to 3.23$)$ $p<0.001$ ), respectfully and were not associated with HIV infection. Alcohol use was strongly associated with food insufficiency $(\mathrm{aOR}=3.23(95 \% \mathrm{Cl} 1.99$ to 5.24) $p<0.001)$. Low $B M I$ was inversely associated with HIV infection $(\mathrm{aOR} \approx 0.5)$ and was not correlated with food insufficiency or dietary diversity.

Conclusions: Rwandan women experienced high rates of food insufficiency and low dietary diversity. Extreme poverty, illiteracy and alcohol use, not HIV infection alone, may contribute to food insufficiency in Rwandan women. Food insufficiency, dietary diversity and low BMI do not correlate with one another; therefore, low

\section{ARTICLE SUMMARY}

Article focus

- What structural determinants are associated with food insufficiency, low dietary diversity and low BMI in HIV-negative and HIV-infected women in Rwanda?

- What is the prevalence of food insufficiency, low dietary diversity and low BMI in HIV-negative and HIV-infected women in Rwanda and are these outcomes correlated with each other?

- Hypotheses

1: Poverty, low literacy status and alcohol use are associated with food insufficiency, low dietary diversity and low BMI.

2: Food insufficiency, low dietary diversity and low BMI are highly prevalent and are correlated with one another.

Key messages

- Food insufficiency and low dietary diversity are highly prevalent ( $46 \%$ and $43 \%$, respectively) and are associated with low income and illiteracy and strongly associated with alcohol use.

- BMI $\left(\mathrm{kg} / \mathrm{m}^{2}\right)$ is not correlated with food insufficiency or dietary diversity.

- Significance: food insufficiency and low dietary diversity, known contributors to poor health, are highly prevalent in HIV-negative and HIV-infected women in Rwanda. Low BMI may not be an adequate screening tool for food insufficiency. Extreme poverty, low literacy and alcohol use may contribute to food insufficiency and low dietary diversity. These structural factors may be useful targets to prevent the adverse health effects of food insufficiency and low dietary diversity.

Strengths and limitations of this study

- Large cohort of HIV-negative and HIV-infected women, very detailed tools used for food insufficiency and dietary diversity

- Cross-sectional design, our measurement of food insufficiency is solely by self-report. 
BMI may not be an adequate screening tool for food insufficiency. Further studies are needed to understand the health effects of not having enough food, low food diversity and low weight in both HIVnegative and HIV-infected women.

\section{INTRODUCTION}

Undernutrition, defined as the condition of people whose food consumption is continuously below a minimum dietary energy requirement for maintaining healthy life, affects over 850 million people worldwide and 200 million adults in Sub-Saharan Africa. ${ }^{1-4}$ The effects of the overlap between undernutrition and HIV infection, which affects over 23 million in Sub-Saharan Africa, are not well understood. ${ }^{5}$ In both HIV-negative and HIV-infected individuals, undernutrition, food insufficiency and low dietary diversity are associated with poor health. ${ }^{5-8}$ Food insufficiency may be caused by structural factors: social, political, economic structures or institutions that affect people's ability to control the conditions of their lives and meet their basic needs. Structural determinants of health include distribution of wealth, power and goods, access to education and schools, access to healthcare and housing and environment conditions. These structural determinants play a major role in health inequities and greatly affect health status. ${ }^{9}$ But structural factors associated with food insufficiency in Sub-Saharan women, and how such factors may be addressed to mitigate food insufficiency in the region is not well studied.

Food insufficiency (lack of adequate food to meet daily needs) is one aspect of food insecurity, a complex phenomenon describing lack of access to sufficient quantity and adequate quality of food, and anxiety in procuring food. ${ }^{2}$ Over half of all households in Rwanda are thought to be food insecure, many of which are headed by women. ${ }^{10}$ Rwanda has a significant number of female-headed households ( $31 \%)$, partly due to the high numbers of genocide-related widows, and $62 \%$ of female-headed households live in poverty compared with $54 \%$ of male-headed households. ${ }^{11}$ Especially in vulnerable populations, such as HIV-infected women, gender disparities may prevent women from having control of family resources and the discretionary income necessary for buying food. ${ }^{12}$

In HIV-infected women, food insufficiency may result in low body mass index (BMI), which adversely affects health outcomes. ${ }^{13}$ In addition, consuming fewer nutritionally distinct food groups (low dietary diversity), which contributes to poor health outcomes in African women and children, ${ }^{7}$ may reinforce malnutrition and eventually result in poor health. ${ }^{14}$ Many African diets consist of a single dominant carbohydrate group, such as cassava, potato or yam, which provides calories that may maintain body weight but often does not provide the micro and macronutrients needed for proper immune function. $^{15}$
In HIV-infected individuals, food insecurity has been associated with low CD4 counts, virologic failure and increased mortality. ${ }^{6}{ }^{8}$ Low BMI $\left(<18.5 \mathrm{~kg} / \mathrm{m}^{2}\right)$ is a strong predictor for mortality in HIV-positive patients starting antiretroviral therapy (ART), with higher mortality in persons who are both food insecure and underweight versus underweight but food secure. ${ }^{8}$ Although poverty is associated with poor health outcomes, income alone does not always reflect the status of someone's 'wealth'. In populations with very low incomes, markers for disposable or discretionary income, defined as income after all essential items are paid for, may be more useful to define an individual's socioeconomic status. These may include access to electricity and ability to buy non-essential items such as alcohol. For women with HIV, it is unclear which structural factors most influence food insecurity and therefore have the greatest impact on health outcomes.

In order to understand structural determinants of food insufficiency and elucidate potential interventions to prevent food insufficiency and malnutrition in HIVnegative and HIV-infected women, we examined the prevalence and socio-demographic associations of food insufficiency, household dietary diversity and low BMI in such women in Rwanda. We were specifically interested in the relationship between poverty, low literacy and alcohol use on food insufficiency, dietary diversity and low BMI. We further examined the relationship between food insufficiency, low BMI and low dietary diversity and whether these three outcomes were correlated with one another in these women.

\section{METHODS}

\section{Population and setting}

The Rwanda Women's Interassociation Study and Assessment (described in detail elsewhere ${ }^{16}$ ) is a prospective observational cohort designed to assess the effectiveness and toxicity of ART in HIV-infected Rwandan women. In 2005, 710 HIV-infected and 226 HIV-uninfected Rwandan women were recruited through community-based women's organisations and HIV clinical care sites. Eligible women were 25 years or older at study entry, willing to give informed consent and were present in Rwanda during the genocide. HIVinfected women were excluded if they had prior history of receiving antiretroviral treatment, except single-dose nevirapine to prevent mother to infant transmission of HIV. Women were compensated 2500 Rwandan francs for each visit. The Rwandan National Ethics Committee and the Institutional Review Board at Montefiore Medical Center approved this study.

At each study visit, participants provided historical information. Trained research assistants collected sociodemographic data at study entry including age, income, literacy level, number of people in households, employment, access to electricity antiretroviral use (for HIV-infected women) and alcohol use. At each visit, participants had a physical examination and 
provided blood specimens for CD4 lymphocyte and complete blood counts. This analysis included 161 HIVnegative and 514 HIV-infected women who completed socio-demographic and nutritional data at the fifth semi-annual visit, between July and December 2007.

\section{Measures}

\section{Primary outcomes}

Food insufficiency was assessed using a single question, 'Do you have enough food?' with the women answering 'usually not' or 'never' classified as food insecure. ${ }^{17} 18$ Household dietary diversity was assessed using a modified Household Dietary Diversity Score (HDDS), a validated tool measuring household food consumption over the previous $24 \mathrm{~h}$, giving one point each for having eaten an item in the following food class (total six points possible: class 1, cereals and roots; class 2, vegetables; class 3, fruits; class 4, meat protein (including meat, eggs, fish); class 5, vegetable protein (including legumes, beans, nuts); class, 6 extras (including oil, fat, sugar, condiments)). Determination of 'low household dietary diversity' is described in detail elsewhere ${ }^{19}$; briefly, the sample was divided into income terciles with the mean HDDS for the lowest income tercile $(\leq 3)$ representing low dietary diversity. ${ }^{15} 19{ }^{20}$ BMI was calculated using weight divided by height-squared $\left(\mathrm{kg} / \mathrm{m}^{2}\right)$. Standing height and weight were measured while the participant was wearing light clothing and no shoes. BMI was dichotomised to $\leq 18.5$ or $>18.5$ for the analysis.

\section{Independent variables}

Income categories were defined as (1) >35 000 Rwandan Francs (\$US58), (2) 35000-10000 and (3) $<10000$ RWF (\$US17) per month. Alcohol use was queried as "Since the last visit, have you had a drink containing alcohol?" and was dichotomised to yes versus no. Literacy was defined as 'can read all, most, some or none', and for the analysis was dichotomised to none versus some, most or all. For HIV-infected women, antiretroviral use at the current visit was assessed by self-report with verification of date of initiation and regimen by tracking cards provided to the participants by providers in the national treatment programme. CD4 counts were determined with an FACS counter (Becton and Dickinson, Immunocytometry Systems, San Jose, California, USA).

\section{Data analysis}

Categorical variables were compared using contingency tables with $\mathrm{p}$ values from exact tests. Univariate logistic regression identified factors associated with food insecurity, low dietary diversity and BMI. Multivariate logistic regression models were built using backward selection with a $\mathrm{p}$ value of 0.05 to stay in the model. Wilcoxon rank sum and kappa statistics assessed relationships between food insecurity, BMI and dietary diversity as continuous variables and as dichotomous variables (food insecurity $=$ answering 'usually not' or 'never' to 'Do you have enough food', HDDS $\leq 3$,
BMI $\leq 18.5), \quad$ respectively. Statistical analysis was performed using SAS (V.9.1.3; SAS Institute Inc.).

\section{RESULTS}

Overall food insufficiency (top row of table 1) was highly prevalent with $46 \%$ of women reporting 'usually not' or 'never' to 'Do you have enough food?' and another $45 \%$ reporting they 'sometimes' did not have enough food (data not shown). Almost half the population reported low dietary diversity (HDDS $<3$ ) and $15 \%$ of women met WHO criteria for malnutrition with a BMI $<18.5 \mathrm{~kg} / \mathrm{m}^{2}$. The percentage of women reporting food insufficiency and low dietary diversity did not differ between HIVnegative and HIV-infected women. The percentage of women with $\mathrm{BMI}<18.5$ was higher in HIV-negative women as compared with HIV-infected women with $\mathrm{CD} 4>350,200-350,<200 \quad(24 \%$, vs $11.8 \%, 12.4 \%$, $14.9 \%, \mathrm{p}=0.004)$, respectively.

Table 1 further breaks the food insecurity outcomes down by participant characteristics. As the numbers in column 2 of table 1 show, the prevalence of poverty was high; $36 \%$ reported a monthly income of $<10 \mathrm{~K}$ Rwandan Francs (FRW) (\$US17). Illiteracy was present in one-quarter of the population and another $40 \%$ reported only reading 'some'. Alcohol use was rare with $13 \%$ reporting they had at least one drink in the last month. Of the HIV-infected women, almost one-third had CD4 counts over 350 and almost $70 \%$ of participants took ART at this visit.

Structural factors associated with food insufficiency included (table 1) low income, with $52.5 \%$ of those with monthly income $<10000$ FRW, $43.6 \%$ of those with monthly income $10000-35000$ FRW and $31.8 \%$ of those with $>35000$ FRW reporting food insufficiency, $\mathrm{p}=0.001$; illiteracy with $57.1 \%$ of those who can not read, $44.5 \%$ of those with some literacy and $34.6 \%$ of those fully literate reporting food insufficiency, $\mathrm{p}=0.0002$, and alcohol use with $68.9 \%$ of users vs $41.2 \%$ of non-users reporting food insufficiency, $\mathrm{p}<0.0001$.

Structural factors associated with low dietary diversity $($ HDDS $<3)$ were; low income with $58.9 \%$ of those with monthly income $<10000$ FRW, $39.3 \%$ with income 10000-35000 FRW, and $16.2 \%$ of those with income $>35000$ FRW having low dietary diversity, $\mathrm{p}<0.0001$; illiteracy with $55.2 \%, 44.4 \%$ and $31.6 \%$ of those with none, some and complete literacy having low dietary diversity, $\mathrm{p}<0.0001$.

Only HIV status had statistically significant associations with BMI. Surprisingly, the association was in the opposite direction expected with $24.2 \%$ of HIV-negative women compared with $11.8 \%-14.9 \%$ of HIV-positive women of all CD4 levels having BMI $<18.5, p=0.004$.

The univariate logistic regression models of table 2 find the same unadjusted associations of structural factors with outcomes just described for table 1 , here we discuss the multivariate modes. In the final stepwise multivariate model (table 2), food insufficiency was independently associated with low income (adjusted 


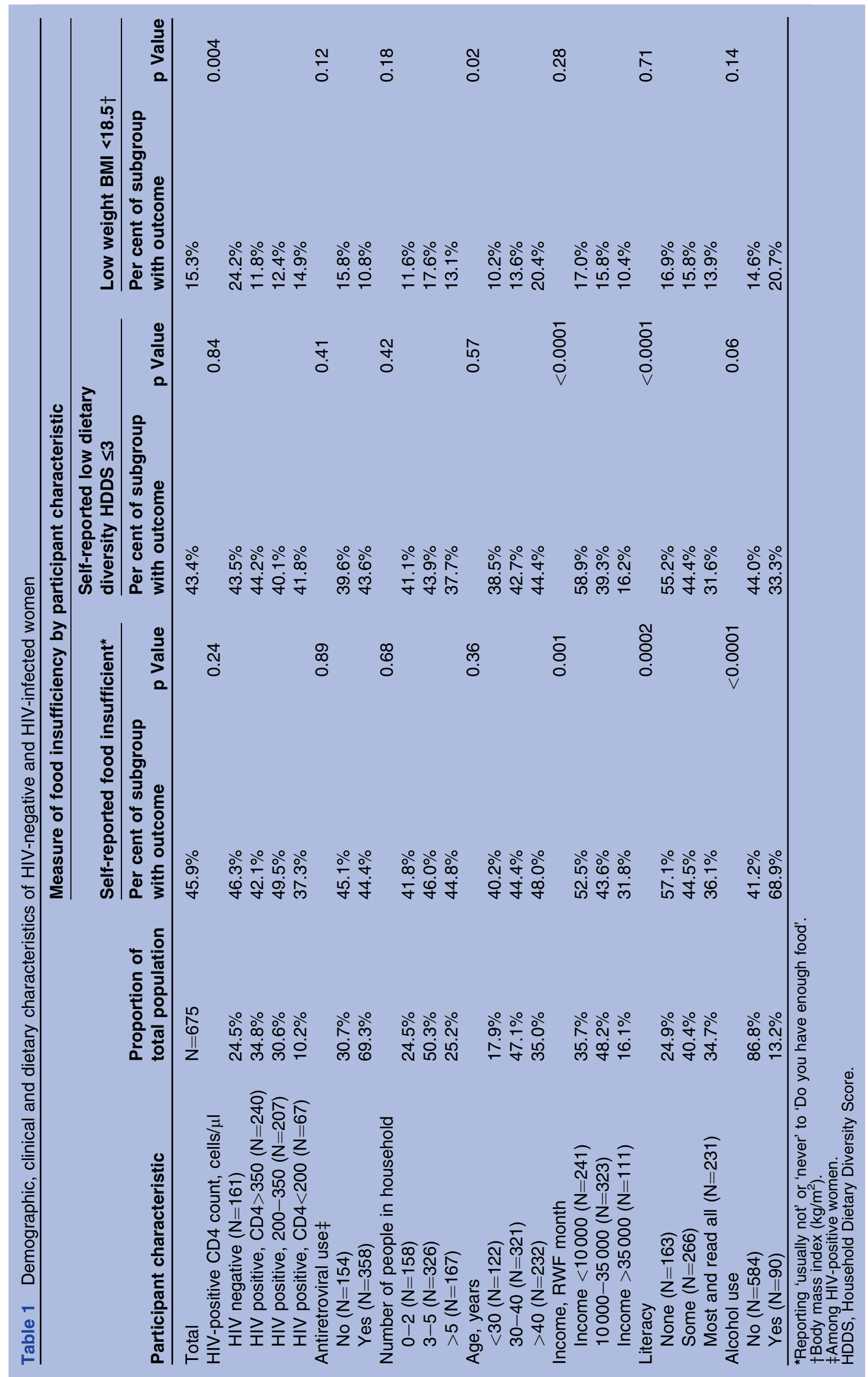




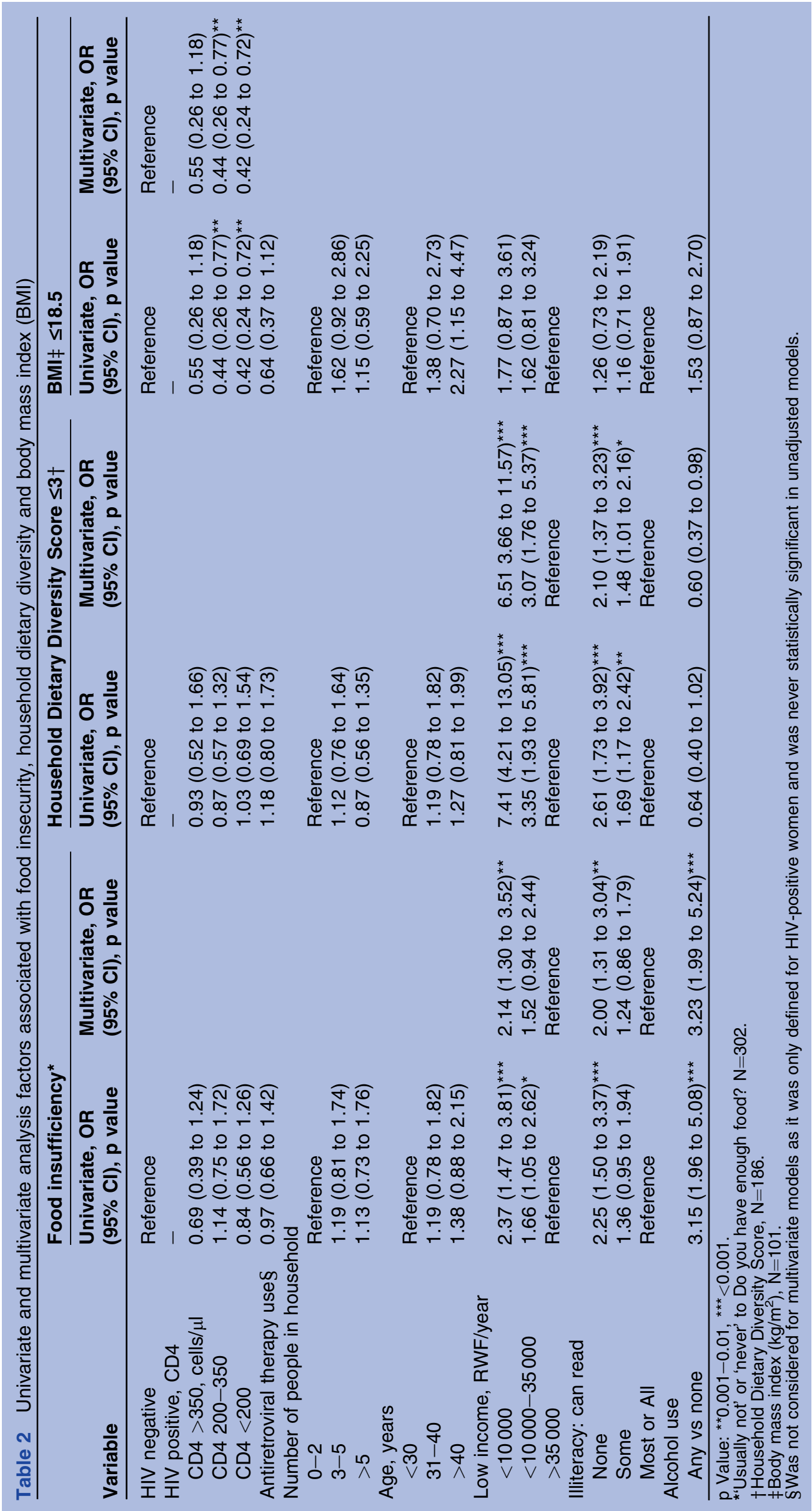


odds ratio (aOR) 2.14, 95\% CI 1.30 to 3.52 for $>35000$ FRW vs $<10000$ FRW) and illiteracy $(\mathrm{aOR}=2.00,95 \% \mathrm{CI}$ 1.31 to 3.04 ) (table 2). Alcohol use (none vs any use) was strongly independently associated with being food insufficient $(\mathrm{aOR}=3.23,95 \%$ CI 1.99 to 5.24). Factors independently associated with low dietary diversity (HDDS <3.0) included low monthly income $(\mathrm{aOR}=6.51$, $95 \%$ CI 3.36 to 11.57 for income $<10000$ vs $>35000$ FRW and $\mathrm{aOR}=3.07,95 \%$ CI 1.76 to 5.37 for income10 000 to $35000 \mathrm{FRW}$ vs $>35000 \mathrm{FRW}$ ) and illiteracy $(\mathrm{aOR}=2.10,95 \% \mathrm{CI} 1.37$ to 3.23$)$. As in table 1 , HIV status had the only independent association with HIV-positive women of all CD4 levels being less likely to have low BMI.

When analysed as continuous variables, no significant correlations were found between self-reported food insufficiency and BMI $(r=-0.05, p=0.29)$. A statistically significant but weak correlation was found between dietary diversity and BMI, $\mathrm{r}=0.09, \mathrm{p}=0.03$, and food insufficiency and dietary diversity, $\mathrm{r}=0.14, \mathrm{p}<0.001$. When analysed as dichotomous variables, no significant correlations were found between food insufficiency and $\mathrm{BMI}$, kappa $(\mathrm{K})=0.02$, dietary diversity and BMI, $\mathrm{K}=0.02$, or food insufficiency and dietary diversity, $\mathrm{K}=0.12$.

\section{DISCUSSION}

While HIV-uninfected and HIV-infected Rwandan women experienced high rates of food insufficiency $(46 \%)$ and low dietary diversity (43\%), only $15 \%$ of the women had low BMI. HIV status did not confer differences except in BMI, where the opposite of what was expected was seen, a higher proportion of HIV-uninfected women had BMI <18.5. Furthermore, neither food insufficiency nor lack of dietary diversity was associated with BMI, suggesting that low BMI in these women was not resulting from food insufficiency alone. Structural factors including low income and illiteracy were associated with food insufficiency, and behavioural factors, such as alcohol use, were associated with low dietary diversity. Our findings highlight three important aspects useful in the relationship between food insufficiency, dietary diversity and BMI in vulnerable populations.

First, few women had low BMI (15\%), while almost half were either food insufficient or had low dietary diversity. A higher percentage of $\mathrm{HIV}$-uninfected women had BMI $<18.5$ as compared with HIV-infected women. This is likely explained by food supplementation programmes provided by community organisations that are available exclusively to the HIV-infected women. These programmes provide additional supply of the staple foods, which may provide enough calories to prevent malnutrition but do not add to dietary diversity or change the perception of not having enough food. Additionally, BMI was not correlated with self-reported food insufficiency or dietary diversity. BMI is often measured in clinical settings and used to monitor people's nutritional status. Because of the known health effects of food insufficiency and low dietary diversity, separate from BMI, ${ }^{6} 7^{14}$ and the lack of correlation between the three outcomes, our results support that BMI should not be considered as a sole marker for food insufficiency in HIV-uninfected or HIV-infected women. The weak association between self-reported food insufficiency and dietary diversity may reflect an inexpensive, abundant single food group, such as potatoes or cassava root, common in Rwanda, that provide a sufficient yet minimally diverse diet. Body weight may be maintainable on a low nutrient density starchy diet that includes suboptimal protein and micronutrient consumption. Additionally, if that single food group is cassava, it provides a much lower protein and micronutrient source than potato, yams or rice.

Second, women who had incomes of $\$ \mathrm{US} 17 /$ month (equal to $10000 \mathrm{RWF} /$ month) were more likely to be food insecure when compared with women whose incomes were \$US60 monthly (equal to $35000 \mathrm{RWF}$ / month). The World Bank defines extreme poverty as \$US1.25/day (\$US37/month) and moderate poverty at \$US2/day (\$US60/month): significant differences exist in health outcomes for these two groups. ${ }^{21}$ This highlights a potentially important target for both ministries of health and international aid organisations and is consistent with the Millennium Development Goals to eradicate poverty. $^{22}$ HIV-negative and HIV-infected women whose income is 1.25 /day may benefit from income supplementation programmes to help prevent food insufficiency and therefore the adverse health effects of food insufficiency and low dietary diversity. ${ }^{23}$ Alternatively, poverty reduction strategies, or job skills training programmes, may be beneficial public health interventions for these women. ${ }^{24-26}$

Alcohol use has known adverse health effects and is a known risk factor for HIV transmission. ${ }^{17}$ Less is known about the relationship with alcohol use and food insufficiency. Alcohol can be used as a marker for disposable income, similar to other luxury items. ${ }^{27}$ In our analysis, alcohol use was rare with only $13 \%$ of the women stating that they had at least one drink in the last month. This shows that casual alcohol drinking is not the social norm in this population of Rwandan women as compared with other nearby countries, such as Uganda, where drinking is considered a socially acceptable activity. The Ugandan Health and Demographic survey found up to onequarter of women reporting drinking in the last month and $18 \%$ stated that they drank alcohol daily. ${ }^{28} 29$ Alcohol use was associated with higher rates of food insufficiency, even when controlled for by income, suggesting it reflected diversion of disposable income from food to alcohol, not just a reflection that more money is available for alcohol purchase. Data on alcohol misuse in Rwandan women are limited, with an estimated national pure alcohol use of 4.31 per capita. ${ }^{30}$ Our data further support the use of alcohol as an important point of intervention to help prevent the adverse health effects of food insufficiency and low dietary diversity. 
Lastly, we found that illiteracy was independently associated with greater food insufficiency and low dietary diversity. This may be because low literacy is an important aspect of 'income-generating capacity', which is critical to ability to obtain relevant dietary diversity and food security. Gender differences in educational and literacy attainment in Rwanda may lead to men procuring non-farm jobs with increased income potential which may increase the numbers of women left to manage the family agricultural plots. ${ }^{31}$ Further studies need to be done to determine if literacy programmes would benefit the level of food security and health status of both HIV-negative and HIV-infected women. Improving land reform laws in Rwanda that strengthen women's positions to own and farm their own land, and empower them with alternative farming techniques, may increase their food security in both urban and rural areas and therefore improve their health. ${ }^{32-34}$

Limitations of our study include its cross-sectional nature, which does not allow us to infer causality. Our measurement of food insufficiency is solely by selfreport. The question, 'Do you have enough food?' does not address the quantity or quality of food or the anxiety surrounding food procurement, although this question has been used in other food insufficiency analyses in Sub-Saharan Africa. ${ }^{17}$ There was no explicit statement that this question would not alter a participant's subsequent eligibility for food aid, which may have introduced response bias. More complete information may be obtained with a different measurement tool, which would address food insecurity (insufficiency quantity, quality or anxiety in procuring food), in addition to food insufficiency (not enough food). ${ }^{18} 20$ A longitudinal study design would be helpful to determine the specific health effects of food insecurity on women over time.

Our findings suggest that extreme poverty, illiteracy and alcohol use are associated with food insufficiency among HIV-infected and HIV-negative women in Rwanda. Addressing these structural factors through income-generating activities, literacy programmes, substance abuse treatment, or perhaps most importantly, renewed health through improved access to ART for HIV-infected women, may help reduce the highly prevalent problem of food insufficiency in the Sub-Saharan region.

\section{Author affiliations}

${ }^{1}$ Department of Medicine, Weill Cornell Medical College, New York, New York, USA

${ }^{2}$ Department of Statistics and Biostatistics, Institute for Health, Health Care Policy and Aging Research, Rutgers University, New Brunswick, New Jersey, USA

${ }^{3}$ Department of Epidemiology and Population Health, Albert Einstein College of Medicine, Bronx, New York, USA

${ }^{4}$ Department of Epidemiology and Community Health, New York Medical

College, Valhalla, New York, USA

${ }^{5}$ Department of Epidemiology and Population Health, Albert Einstein College of Medicine, Bronx, New York, USA

${ }^{6}$ Women's Equity in Access to Care and Treatment, Kigali, Rwanda

${ }^{7}$ Department of Medicine, John Stroger (formerly Cook County) Hospital and Rush University, Chicago, Illinois, USA
${ }^{8}$ Departments of Medicine and Epidemiology and Population Health, Montefiore Medical Center and Albert Einstein College of Medicine, Bronx, New York, USA

Acknowledgements We thank the patients and staff of Rwanda Women's Interassociation Study and Assessment.

Contributors KA, EM and CJS-I designed the research. NS, QS, DH, CJS-I and KA analysed the data. NS wrote the paper with input from all authors. NS had primary responsibility for the final content. All authors read and approved the final manuscript.

Funding This project has been funded by NIH/NIDA/NIAID/NCI grant 5U01Al035004-16 (KA).

\section{Competing interests None.}

Ethics approval Ethics approval was provided by Rwandan National Ethics Committee and the Institutional Review Board at Montefiore Medical Center.

Provenance and peer review Not commissioned; externally peer reviewed.

Data sharing statement There are no additional data available.

\section{REFERENCES}

1. Reaching sustainable food security for all by 2020: getting the priorities and responsibilities right. Washington, DC: International Food Policy Research Institute, 2002.

2. Bickel G, Nord M, Price C, et al. Guide to Measuring Household Food Security. Alexandria VA: U.S. Department of Agriculture, Food and Nutrition Service, 2000.

3. Women in Agriculture: Closing the Gender Gap For Development. Rome: Food and Agriculture Organization, 2011.

4. Shapouri SRS, Peters M, Tandon S, et al. International Food Security Assessment 2011-21. Washington, DC: Economic Research Service/ US Department of Agriculture, 2011.

5. UNAIDS. Global Report Fact Sheet. Sub Saharan Africa Geneva: Joint United Nations Programme on HIV/AIDS, 2011.

6. Weiser SD, Frongillo E, Ragland K, et al. Food insecurity is associated with incomplete HIV RNA suppression among homeless and marginally housed HIV-infected individuals in San Francisco. $J$ Gen Inter Med 2009;24:14-20.

7. Mpontshane N, Van den Broeck J, Chhagan M, et al. HIV infection is associated with Decreased dietary diversity in South African children. J Nutr 2008;138:1705-11.

8. Weiser SD, Fernandes KA, Brandson EK, et al. The association between food insecurity and mortality among HIV-infected individuals on HAART. J Acquir Immune Defic Syndr 2009;52:342-9.

9. Closing the gap in a generation: Health Equity through Action on the Social Determinants of Health in Final Report of the Commission on Social Determinants of Health. Geneva: World Health Organization, 2008.

10. Millennium Development Goals. Towards Sustainable Social and Economic Growth. First Edition. Country Report 2007. Kigali: The national Institute of statistics of Rwanda (NISR), 2007.

11. Abbott P, Rwirahira J. Millennium Development Goals Rwanda Country Report 2010. Kigali: UNDP, 2010.

12. Karp RJ, Chang C, Meyers AF. The appearance of discretionary income: influence on the prevalence of under- and over-nutrition. Int $J$ Equity Health 2005;4:10.

13. Jee SH, Sull JW, Park J, et al. Body-Mass index and mortality in Korean men and women. New Engl J Med 2006;355:779-87.

14. Savy M, Martin-Prevel $\mathrm{Y}$, Sawadogo $\mathrm{P}$, et al. Use of variety/diversity scores for diet quality measurement: relation with nutritional status of women in a rural area in Burkina Faso. Eur J Clin Nutr 2005;59:703-16.

15. Becquey E, Martin-Prevel Y. Micronutrient Adequacy of Women's diet in urban Burkina Faso is low. J Nutr 2010;140:2079S-85S.

16. Cohen M, Fabri M, Cai X, et al. Prevalence and predictors of Posttraumatic Stress Disorder and Depression in HIV-Infected and at-risk Rwandan women. J Womens Health 2009;18:1783-91.

17. Weiser SD, Leiter K, Bangsberg DR, et al. Food insufficiency is associated with high-risk sexual behavior among women in Botswana and Swaziland. PLoS Med 2007;4:1589-97.

18. Coates J, Swindale A, Bilinsky P. Household Food Insecurity Access Scale (HFIAS) for Measurement of Household Food Access: Indicator Guide in Food and Nutrition Technical Assistance Project. Washington, DC: Academy for Educational Development, 2007.

19. Swindale A, Bilinsky P. Household Dietary Diversity Score (HDDS) for Measurement of Household Food Access: Indicator Guide in Food and Nutrition Technical Assistance Project (FANTA). Washington, DC: Academy for Educational Development, 2006. 
20. Becquey E, Martin-Prevel Y, Traissac $\mathrm{P}$, et al. The household food insecurity access Scale and an index-Member dietary diversity Score contribute Valid and Complementary information on household food insecurity in an urban West-African Setting. J Nutr 2010;140:2233-40.

21. The World Bank. Poverty. The World Bank, 2010. http://data. worldbank.org/topic/poverty (accessed 31 Mar 2012).

22. The Millennium Development Goals Report. New York: Department of Economic and Social Affairs, 2009.

23. Braine T. Reaching Mexico's poorest. Bull World Health Organ 2006;84:592-3.

24. Laterveer L, Niessen L, Yazbeck AS. Pro-poor health policies in poverty reduction strategies. Health Policy Plan 2003:18:138-45.

25. Nguyen P, Bich Hanh D, Lavergne MR, et al. The effect of a poverty reduction policy and service quality standards on commune-leve primary health care utilization in Thai Nguyen Province, Vietnam. Health Policy Plan 2010;25:262-71.

26. Dodd R, Hinshelwood E, Harvey C. Poverty Reduction Strategy Papers: Their Significance for health in Second Synthesis report. Geneva: World Health Organization, 2004.

27. Citro CF, Michael RT, eds. Measuring Poverty: A New Approach Washington, DC: National Academy Press, 1995.
28. UBOS \& ORC Macro. Uganda Demographic and Health Survey 2000-2001. Calverton, Maryland, USA: Uganda Bureau of Statistics and ORC Macro, 2001.

29. http://www.who.int/substance_abuse/publications/alcohol_gender_ drinking _problem.pdf\#page $=200$ (assessed 2 Apr 2012).

30. http://www.who.int/substance_abuse/publications/en/rwanda.pdf (accessed 20 Nov 2011).

31. World Bank. Education in Rwanda: Rebalancing Resources To Accelerate Post-Conflict Development And Poverty Reduction, in AWorld Bank Country Study. The International Bank for Reconstruction and Development. Washington DC: The World Bank, 2004

32. Rao N. Land rights, gender equality and household food security: Exploring the conceptual links in the case of India. Food Policy 2006;31:180-93.

33. World Bank. World Development Report 2008: Agriculture for Development. The international Bank for Reconstruction and Development/The World Bank, 2008.

34. Maxwell D. Alternative food security strategy: a household analysis of urban agriculture in Kampala. World Dev 1995;23 1669-81. 